\title{
Generating nonisospectral integrable hierarchies via a new scheme
}

\author{
Haifeng Wang ${ }^{1}$ and Yufeng Zhang ${ }^{1 *}$
}

"Correspondence:

zhangyfcumt@163.com

'School of Mathematics, China

University of Mining and

Technology, Xuzhou, P.R. China

\section{Springer}

\begin{abstract}
In the paper, an efficient and straightforward method for generating nonisospectral integrable hierarchies is introduced. It follows that we consider the application related to Lie algebra gl(3) based on the method. Then, we derive a nonisospectral integrable hierarchy whose some new symmetries are also investigated. In addition, a few conserved quantities of the nonisospectral integrable hierarchies are also obtained.
\end{abstract}

PACS Codes: $05.45 . Y v ; 02.30 . J r ; 02.30 .1 \mathrm{k}$

Keywords: Nonisospectral integrable hierarchy; Lie algebras; Hamiltonian structure; Symmetries

\section{Introduction}

We know that one approach for generating integrable systems was proposed by Magri [1], which was called the Lax-pair method [2, 3]. Based on it, Tu [4] proposed a method for generating integrable Hamiltonian hierarchies, which was called the Tu scheme by $\mathrm{Ma}$ [5]. Through making use of the Tu scheme, some integrable systems and the corresponding Hamiltonian structures as well as other properties were obtained, such as the works in [6-10]. It is well known that many different methods for generating isospectral integrable equations have been proposed [11-15]. However, as nonisospectral integrable equations are concerned, fewer works have been presented, as far as we know. Ma [16, 17] applied Lax equations to work out some nonisospectral integrable hierarchy under the case of $\lambda_{t}=\lambda^{n}(n>0)$. Qiao [18] adopted the Lenard series method to obtain some nonisospectral integrable hierarchies under the case $\lambda_{t}=\lambda^{m+1} M$. The aim of this paper is to apply an efficient scheme to generate nonisospectral integrable hierarchies of evolution equations under the case where $\lambda_{t}=\sum_{j=0}^{n} k_{j}(t) \lambda^{n-j}$. Obviously, this case is a generalized expression for the case $\lambda_{t}=\lambda^{n}[19,20]$. Under obtaining nonisospectral integrable systems, some of their properties, including Darboux transformations, exact solutions, and so on, could be studied [21-26]. We first recall some fundamental facts.

Let $G$ be a finite-dimensional Lie algebra over the complex set $C, \widetilde{G}=G \otimes C\left[\lambda, \lambda^{-1}\right]$ be the corresponding loop algebra, where $C\left[\lambda, \lambda^{-1}\right]$ stands for a set of Laurent polynomials in the parameter $\lambda$. Suppose that $\left\{e_{1}, \ldots, e_{p}\right\}$ is a basis of $G$, then the basis of the loop algebra $\widetilde{G}$ can be chosen as $\left\{e_{1}(n), \ldots, e_{p}(n)\right\}$, where $e_{i}(n)=e_{i} \lambda^{N_{i} n}, N_{i}=1,2, \ldots, n \in Z$.

(c) The Author(s) 2020. This article is licensed under a Creative Commons Attribution 4.0 International License, which permits use, sharing, adaptation, distribution and reproduction in any medium or format, as long as you give appropriate credit to the original author(s) and the source, provide a link to the Creative Commons licence, and indicate if changes were made. The images or other third party material in this article are included in the article's Creative Commons licence, unless indicated otherwise in a credit line to the material. If material is not included in the article's Creative Commons licence and your intended use is not permitted by statutory regulation or exceeds the permitted use, you will need to obtain permission directly from the copyright holder. To view a copy of this licence, visit http://creativecommons.org/licenses/by/4.0/. 
Definition 1 One basis element $R \in \widetilde{G}$ is called pseudoregular if the following conditions hold:

(1) $\widetilde{G}=\operatorname{Ker} \operatorname{ad} R \oplus \operatorname{Im} \operatorname{ad} R$,

(2) $\operatorname{ker} \operatorname{ad} R$ is commutative, where

$\operatorname{Ker} \operatorname{ad} R=\{x \mid x \in \widetilde{G},[x, R]=0\}, \operatorname{Imad} R=\{x \mid \exists y \in \widetilde{G}, x=[y, R]\}$.

Definition 2 For any basis element $e_{i}(n)(i=1,2, \ldots, p)$, we define its gradation by

$$
\operatorname{deg}\left(e_{i}(n)\right)=N_{i} n
$$

Obviously, for $\forall g \in \widetilde{G}, g$ can be expressed by $g=\sum_{n} k_{n} e_{i}(n)=: \sum_{n} g_{n}, k_{n}$ are constants. We can decompose $g$ into two parts as follows:

$$
g_{+}=\sum_{n \geq \mu} g_{n}, \quad g_{-}=\sum_{n<\mu} g_{n}
$$

and call $g_{+}$the positive part of $g, \mu \in Z$ is some chosen integer.

In the following, the steps for generating nonisospectral integrable hierarchies of evolution equations are presented.

Step 1: By using the loop algebra $\widetilde{G}$, we introduce the spectral problems

$$
\begin{aligned}
& \psi_{x}=U \psi, \quad U=R+u_{1} e_{1}(n)+\cdots+u_{q} e_{q}(n), \\
& \psi_{t}=V \psi, \quad V=A_{1} e_{1}(n)+\cdots+A_{p} e_{p}(n), \\
& \lambda_{t}=\sum_{i \geq 0} k_{i}(t) \lambda^{-N_{i} i},
\end{aligned}
$$

where the potential functions $u_{1}, \ldots, u_{q} \in S$ (the Schwartz space), and $R(n), e_{1}(n), \ldots$, $e_{p}(n) \in \widetilde{G}$ satisfy that

(a) $R, e_{1}, \ldots, e_{p}$ are linear independent,

(b) $R$ is pseudoregular,

(c) $\operatorname{deg}(R(n)) \geq \operatorname{deg}\left(e_{i}(n)\right), i=1,2, \ldots, p$.

Step 2: Solving the following stationary zero curvature equation for $A_{i}, i=1,2, \ldots, p$ :

$$
V_{x}=\frac{\partial U}{\partial \lambda} \lambda_{t}+[U, V] .
$$

It follows that one can get the compatibility condition of (2) and (3)

$$
\frac{\partial U}{\partial u} u_{t}+\frac{\partial U}{\partial \lambda} \lambda_{t}-V_{x}+[U, V]=0 .
$$

Equation (6) can be broken down into

$$
-V_{+, x}^{(n)}+\frac{\partial U}{\partial \lambda} \lambda_{t,+}^{(n)}+\left[U, V_{+}^{(n)}\right]=V_{-, x}^{(n)}-\frac{\partial U}{\partial \lambda} \lambda_{t,-}^{(n)}-\left[U, V_{-}^{(n)}\right],
$$

where

$$
\lambda_{t,+}^{(m)}=\lambda^{N_{i} m} \lambda_{t}-\lambda_{t,-}^{(m)}=\sum_{i=\mu}^{m} k_{i}(t) \lambda^{N_{i} m-N_{i} i+x}, \quad x=0,1, \ldots, N_{i}-1 ; m<n .
$$


Step 3: Choose $\triangle_{n} \in \widetilde{G}$ so that

$$
\begin{aligned}
& V^{(n)}=\left(\lambda^{N_{i}^{n}} V\right)_{+}+\triangle_{n}=: V_{+}^{(n)}+\triangle_{n}, \\
& -V_{x}^{(n)}+\frac{\partial U}{\partial \lambda} \lambda_{t,+}^{(n)}+\left[U, V^{(n)}\right]=B_{1} e_{1}+\cdots+B_{q} e_{q},
\end{aligned}
$$

where $B_{i}(i=1,2, \ldots, q) \in C$.

Step 4: The nonisospectral integrable hierarchies of evolution equations could be deduced via the nonisospectral zero curvature equation

$$
\frac{\partial U}{\partial u} u_{t}+\frac{\partial U}{\partial \lambda} \lambda_{t,+}^{(n)}-V_{x}^{(n)}+\left[U, V^{(n)}\right]=0 .
$$

Step 5: The Hamiltonian structures of hierarchies (8) are sought out according to the trace identity given by Tu [4].

\section{A nonisospectral integrable hierarchy of evolution equations}

A basis of the Lie algebras $\mathrm{gl}(3)$ is given by

$$
\operatorname{gl}(3)=\operatorname{span}\{h, e, f\}
$$

with

$$
h=\left(\begin{array}{ccc}
0 & 0 & -1 \\
0 & 0 & 0 \\
1 & 0 & 0
\end{array}\right), \quad e=\left(\begin{array}{ccc}
0 & 0 & 0 \\
0 & 0 & -1 \\
0 & 1 & 0
\end{array}\right), \quad f=\left(\begin{array}{ccc}
0 & -1 & 0 \\
1 & 0 & 0 \\
0 & 0 & 0
\end{array}\right) .
$$

And the corresponding loop algebra is taken by

$$
\widetilde{\mathrm{gl}}(3)=\operatorname{span}\{h(n), e(n), f(n)\},
$$

where $h(n)=h \lambda^{2 n}, e(n)=e \lambda^{2 n-1}, f(n)=f \lambda^{2 n-1}$.

After simple calculations, one can find

$$
\begin{aligned}
& {[h(n), e(m)]=f \lambda^{2 n+2 m-1}=f(m+n), \quad[h(n), f(m)]=-e(m+n),} \\
& {[e(n), f(m)]=h(m+n-1), \quad m, n \in Z,}
\end{aligned}
$$

where the gradations of $h(n), e(n)$, and $f(n)$ are given by

$$
\operatorname{deg} h(n)=2 n, \quad \operatorname{deg} e(n)=2 n-1, \quad \operatorname{deg} f(n)=2 n-1, \quad n \in Z .
$$

We consider the following nonisospectral problems based on $\widetilde{\mathrm{gl}}(3)$ :

$$
\psi_{x}=U \psi, \quad U=-\bar{i} h(1)+q e(1)+r f(1)=\left(\begin{array}{ccc}
0 & -r \lambda & \bar{i} \lambda^{2} \\
r \lambda & 0 & -q \lambda \\
-\bar{i} \lambda^{2} & q \lambda & 0
\end{array}\right)
$$




$$
\psi_{t}=V \psi, \quad V=a h(0)+b e(1)+c f(1)=\left(\begin{array}{ccc}
0 & -c \lambda & -a \\
c \lambda & 0 & -b \lambda \\
a & b \lambda & 0
\end{array}\right)
$$

where $\bar{i}^{2}=-1, a=\sum_{i \geq 0} a_{i} \lambda^{-2 i}, b=\sum_{i \geq 0} b_{i} \lambda^{-2 i}, c=\sum_{i \geq 0} c_{i} \lambda^{-2 i}$.

It follows that we obtain

$$
\begin{aligned}
\frac{\partial U}{\partial \lambda} \lambda_{t} & =\left(\begin{array}{ccc}
0 & -r & -2 \bar{i} \lambda \\
r & 0 & -q \\
-2 \bar{i} \lambda & q & 0
\end{array}\right) \sum_{i \geq 0} k_{i}(t) \lambda^{-2 i+1} \\
& =\sum_{i \geq 0} k_{i}(t)[-2 \bar{i} h(1-i)+q e(1-i)+r f(1-i)] .
\end{aligned}
$$

Furthermore, the following equation can be derived by taking $\lambda_{t}=\sum_{i \geq 0} k_{i}(t) \lambda^{1-2 i}$ with Eq. (6):

$$
\left\{\begin{array}{l}
a_{i x}=q c_{i+1}-r b_{i+1}-2 \bar{i} k_{i+1}(t) \\
b_{i x}=\bar{i} c_{i+1}+r a_{i}+k_{i}(t) q \\
c_{i x}=-\bar{i} b_{i+1}-q a_{i}+k_{i}(t) r
\end{array}\right.
$$

that is,

$$
\left\{\begin{array}{l}
a_{i x}=-\bar{i}\left(q b_{i x}+r c_{i x}-q^{2} k_{i}(t)-r^{2} k_{i}(t)+2 k_{i+1}(t)\right) \\
c_{i+1}=\bar{i}\left(-b_{i x}+r a_{i}+q k_{i}(t)\right) \\
b_{i+1}=\bar{i}\left(c_{i x}+q a_{i}-r k_{i}(t)\right)
\end{array}\right.
$$

In terms of Eq. (12), we take the initial values

$$
b_{0}=k_{0} \partial^{-1} q, \quad c_{0}=k_{0} \partial^{-1} r,
$$

and then one has

$$
a_{0}=-2 \bar{i} k_{1}(t) x+\beta_{0}(t)
$$

where $\beta_{0}(t)=0$ is an integral constant. From (12), we deduce that

$$
\begin{aligned}
& b_{1}=2 k_{1}(t) q x, \quad c_{1}=2 k_{1}(t) r x, \\
& a_{1}=-\bar{i} k_{1}(t) x\left(q^{2}+r^{2}\right)-2 \bar{i} k_{2}(t) x+\beta_{1}(t), \\
& b_{2}=\bar{i} k_{1}(t)\left(r+2 x r_{x}\right)+q x\left(k_{1}(t) q^{2}+k_{1}(t) r^{2}+2 k_{2}(t)\right), \\
& c_{2}=-\bar{i} k_{1}(t)\left(q+2 x q_{x}\right)+r x\left(k_{1}(t) q^{2}+k_{1}(t) r^{2}+2 k_{2}(t)\right), \\
& \cdots,
\end{aligned}
$$

where $\beta_{1}(t)=0$ is an integral constant. Denote that

$$
V_{+}^{(n)}=\sum_{i=0}^{n}\left(a_{i} h(n-i)+b_{i} e(n+1-i)+c_{i} f(n+1-i)\right)
$$




$$
\begin{aligned}
& V_{-}^{(n)}=\sum_{i=n+1}^{\infty}\left(a_{i} h(n-i)+b_{i} e(n+1-i)+c_{i} f(n+1-i)\right), \\
& \lambda_{t,+}^{(n)}=\sum_{i=0}^{n} K_{i}(t) \lambda^{2 n-2 i+1}, \quad \lambda_{t,-}^{(n)}=\sum_{i=n+1}^{\infty} K_{i}(t) \lambda^{2 n-2 i+1} .
\end{aligned}
$$

In what follows, the gradations of the left-hand side of (7) can be obtained by using (1), (9), and (10)

$$
\begin{aligned}
& \operatorname{deg} V_{+}^{(n)}=:(0,1,1) \geq 0, \quad \operatorname{deg} \frac{\partial U}{\partial \lambda} \lambda_{t,+}^{(n)}=:(2,1,1) \geq 1, \\
& \operatorname{deg}\left(\left[U, V_{+}^{(n)}\right]\right)=:(2,1,1 ; 0,1,1) \geq 1,
\end{aligned}
$$

which indicates that the minimum gradation of the left-hand side of (7) is zero. Additionally, we also obtain the gradations of the right-hand side of (7) as follows:

$$
\begin{aligned}
& \operatorname{deg} V_{-}^{(n)}=:(-2,-1,-1) \leq-1, \quad \operatorname{deg} \frac{\partial U}{\partial \lambda} \lambda_{t,-}^{(n)}=:(0,-1,-1) \leq 0, \\
& \operatorname{deg}\left(\left[U, V_{-}^{(n)}\right]\right)=:(2,1,1 ;-2,-1,-1) \leq 1
\end{aligned}
$$

which means the maximum gradation of the right-hand side of (7) is 1 . Thus, we further infer the following equation by taking these terms which have the gradations 0 and 1 :

$$
\begin{aligned}
& V_{-, x}^{(n)}-\frac{\partial U}{\partial \lambda} \lambda_{t,-}^{(n)}-\left[U, V_{-}^{(n)}\right] \\
& \quad=\bar{i} b_{n+1} f(1)-\bar{i} c_{n+1} e(1)-q c_{n+1} h(0)+r b_{n+1} h(0)+2 \bar{i} K_{n+1}(t) h(0),
\end{aligned}
$$

that is,

$$
\begin{aligned}
- & V_{+, x}^{(n)}+\frac{\partial U}{\partial \lambda} \lambda_{t,+}^{(n)}+\left[U, V_{+}^{(n)}\right] \\
& =\bar{i} b_{n+1} f(1)-\bar{i} c_{n+1} e(1)-q c_{n+1} h(0)+r b_{n+1} h(0)+2 \bar{i} K_{n+1}(t) h(0) .
\end{aligned}
$$

In order to obtain the nonisospectral integrable hierarchies, we take the modified term $\triangle_{n}=-a_{n} h(0)$ so that for $V^{(n)}=V_{+}^{(n)}-a_{n} h(0)$, we have from (13) that

$$
-V_{x}^{(n)}+\frac{\partial U}{\partial \lambda} \lambda_{t,+}^{(n)}+\left[U, V^{(n)}\right]=\left(-\bar{i} c_{n+1}-r a_{n}\right) e(1)+\left(\bar{i} b_{n+1}+q a_{n}\right) f(1) .
$$

Therefore, the nonisospectral integrable hierarchy is derived by Eq. (8) as follows:

$$
\begin{aligned}
u_{t_{n}} & =\left(\begin{array}{l}
q \\
r
\end{array}\right)_{t_{n}}=\left(\begin{array}{l}
-\bar{i} c_{n+1}-r a_{n} \\
\bar{i} b_{n+1}+q a_{n}
\end{array}\right)=\left(\begin{array}{l}
b_{n x}-K_{n}(t) q \\
c_{n x}-K_{n}(t) r
\end{array}\right) \\
& =\left(\begin{array}{ll}
0 & \partial \\
\partial & 0
\end{array}\right)\left(\begin{array}{l}
c_{n} \\
b_{n}
\end{array}\right)-K_{n}(t)\left(\begin{array}{l}
q \\
r
\end{array}\right) \\
& =: J_{1}\left(\begin{array}{l}
c_{n} \\
b_{n}
\end{array}\right)-K_{n}(t)\left(\begin{array}{l}
q \\
r
\end{array}\right),
\end{aligned}
$$


or

$$
\begin{aligned}
u_{t_{n}} & =\left(\begin{array}{l}
q \\
r
\end{array}\right)_{t_{n}}=\left(\begin{array}{c}
-r \partial^{-1} r b_{n+1}+\left(\bar{i}+r \partial^{-1} q\right) c_{n+1}-2 \bar{i} r K_{n+1}(t) x \\
-q \partial^{-1} q c_{n+1}+\left(-\bar{i}+q \partial^{-1} r\right) b_{n+1}+2 \bar{i} q K_{n+1}(t) x
\end{array}\right) \\
& =\left(\begin{array}{cc}
\bar{i}+r \partial^{-1} q & -r \partial^{-1} r \\
-q \partial^{-1} q & -\bar{i}+q \partial^{-1} r
\end{array}\right)\left(\begin{array}{c}
c_{n+1} \\
b_{n+1}
\end{array}\right)+2 \bar{i} K_{n+1}(t) x\left(\begin{array}{c}
-r \\
q
\end{array}\right) \\
& =: J_{2}\left(\begin{array}{c}
c_{n+1} \\
b_{n+1}
\end{array}\right)+2 \bar{i} K_{n+1}(t) x\left(\begin{array}{c}
-r \\
q
\end{array}\right),
\end{aligned}
$$

where

$$
J_{1}=\left(\begin{array}{ll}
0 & \partial \\
\partial & 0
\end{array}\right), \quad J_{2}=\left(\begin{array}{cc}
\bar{i}+r \partial^{-1} q & -r \partial^{-1} r \\
-q \partial^{-1} q & -\bar{i}+q \partial^{-1} r
\end{array}\right)
$$

Based on (12), one has

$$
\begin{aligned}
\left(\begin{array}{l}
c_{n+1} \\
b_{n+1}
\end{array}\right)= & \left(\begin{array}{cc}
r \partial^{-1} r \partial & -\bar{i} \partial+r \partial^{-1} q \partial \\
\bar{i} \partial+q \partial^{-1} r \partial & q \partial^{-1} q \partial
\end{array}\right)\left(\begin{array}{l}
c_{n} \\
b_{n}
\end{array}\right) \\
& +K_{n}(t)\left(\begin{array}{l}
-r \partial^{-1}\left(q^{2}+r^{2}\right)+\bar{i} q \\
-q \partial^{-1}\left(q^{2}+r^{2}\right)-\bar{i} r
\end{array}\right)+2 K_{n+1}(t) x\left(\begin{array}{l}
r \\
q
\end{array}\right) \\
= & L\left(\begin{array}{l}
c_{n} \\
b_{n}
\end{array}\right)+K_{n}(t) Q+2 K_{n+1}(t) x R,
\end{aligned}
$$

where

$$
L=\left(\begin{array}{cc}
r \partial^{-1} r \partial & -\bar{i} \partial+r \partial^{-1} q \partial \\
\bar{i} \partial+q \partial^{-1} r \partial & q \partial^{-1} q \partial
\end{array}\right), \quad Q=\left(\begin{array}{c}
-r \partial^{-1}\left(q^{2}+r^{2}\right)+\bar{i} q \\
-q \partial^{-1}\left(q^{2}+r^{2}\right)-\bar{i} r
\end{array}\right), \quad R=\left(\begin{array}{l}
r \\
q
\end{array}\right) .
$$

Hence, (14) can be written as

$$
\begin{aligned}
u_{t_{n}} & =\left(\begin{array}{c}
q \\
r
\end{array}\right)_{t_{n}} \\
& =J_{1} L^{n}\left(\begin{array}{l}
K_{0} \partial^{-1} r \\
K_{0} \partial^{-1} q
\end{array}\right)+J_{1} \sum_{i=0}^{n-1}\left(L^{i} K_{n-1-i}(t) Q\right)+2 J_{1} \sum_{i=0}^{n-1} L^{i} K_{n-i}(t) x R-K_{n}(t)\left(\begin{array}{l}
q \\
r
\end{array}\right) \\
& =\Phi^{n} K_{0}\left(\begin{array}{l}
q \\
r
\end{array}\right)+\sum_{i=0}^{n-1} \Phi^{i} J_{1} K_{n-1-i}(t) Q+2 \sum_{i=0}^{n-1} K_{n-i}(t) \Phi^{i} \partial\left(\begin{array}{l}
x q \\
x r
\end{array}\right)-K_{n}(t)\left(\begin{array}{l}
q \\
r
\end{array}\right),
\end{aligned}
$$

where

$$
\Phi=J_{1} L J_{1}^{-1}=\left(\begin{array}{cc}
q_{x} \partial^{-1} q+q^{2} & \bar{i} \partial+q_{x} \partial^{-1} r+q r \\
-\bar{i} \partial+r_{x} \partial^{-1} q+q r & r_{x} \partial^{-1} r+r^{2}
\end{array}\right) \text {. }
$$


When $n=1$, the nonisospectral integrable hierarchy (17) becomes

$$
\left\{\begin{array}{l}
q_{t}=2 K_{1}(q x)_{x}+K_{1} q \\
r_{t}=2 K_{1}(r x)_{x}+K_{1} r
\end{array}\right.
$$

When $n=2$, the nonisospectral integrable hierarchy (17) reduces to

$$
\left\{\begin{array}{l}
q_{t}=K_{1}\left(q^{3} x+q r^{2} x+\bar{i} r+2 \bar{i} r_{x} x\right)_{x}+2 K_{2}(q x)_{x}-K_{2} q, \\
r_{t}=K_{1}\left(r^{3} x+r q^{2} x-\bar{i} q-2 \bar{i} q_{x} x\right)_{x}+2 K_{2}(r x)_{x}-K_{2} r .
\end{array}\right.
$$

Additionally, we focus on a format of Hamiltonian construction of hierarchy (17) via the trace identity proposed by Tu [4]. Denote the trace of the square matrices $A$ and $B$ by $\langle A, B\rangle=\operatorname{tr}(A B)$.

Equation (9) and Eq. (10) admit that

$$
\left\langle V, \frac{\partial U}{\partial q}\right\rangle=-2 b \lambda^{2}, \quad\left\langle V, \frac{\partial U}{\partial r}\right\rangle=-2 c \lambda^{2}, \quad\left\langle V, \frac{\partial U}{\partial \lambda}\right\rangle=-2 c r \lambda+4 \bar{i} a \lambda-2 b q \lambda,
$$

which can be substituted into the trace identity to get

$$
\begin{aligned}
& \frac{\delta}{\delta u}\left(\left\langle V, \frac{\partial U}{\partial \lambda}\right\rangle\right)=\lambda^{-\gamma} \frac{\partial}{\partial \lambda} \lambda^{\gamma}\left(\begin{array}{c}
\left\langle V, \frac{\partial U}{\partial q}\right\rangle \\
\left\langle V, \frac{\partial U}{\partial r}\right\rangle
\end{array}\right), \\
& \frac{\delta}{\delta u}(-2 c r \lambda+4 \bar{i} a \lambda-2 b q \lambda)=\lambda^{-\gamma} \frac{\partial}{\partial \lambda}\left(\begin{array}{l}
-2 b \lambda^{2+\gamma} \\
-2 c \lambda^{2+\gamma}
\end{array}\right) .
\end{aligned}
$$

It follows that one can get the following equation by comparing the two sides of the above formula:

$$
\frac{\delta}{\delta u}\left(4 \bar{i} a_{n}-2 q b_{n}-2 r c_{n}\right)=-2(2-2 n+\gamma)\left(\begin{array}{l}
b_{n} \\
c_{n}
\end{array}\right) .
$$

One can find $\gamma=0$ via substituting the initial values of (12) into (22), and then we obtain

$$
\left(\begin{array}{l}
b_{n} \\
c_{n}
\end{array}\right)=\frac{\delta H_{n}}{\delta u}=\left(\begin{array}{ll}
0 & 1 \\
1 & 0
\end{array}\right)\left(\begin{array}{l}
c_{n} \\
b_{n}
\end{array}\right)=: M_{1}\left(\begin{array}{l}
c_{n} \\
b_{n}
\end{array}\right)
$$

where

$$
H_{n}=\frac{2 \bar{i} a_{n}-q b_{n}-r c_{n}}{2 n-2}, \quad M_{1}^{-1}=M_{1}=\left(\begin{array}{ll}
0 & 1 \\
1 & 0
\end{array}\right) .
$$

Hence, hierarchies (14) and (15) can be written as

$$
u_{t_{n}}=\left(\begin{array}{l}
q \\
r
\end{array}\right)_{t_{n}}=J_{1} M_{1} \frac{\delta H_{n}}{\delta u}-K_{n}(t)\left(\begin{array}{l}
q \\
r
\end{array}\right)=J_{2} M_{1} \frac{\delta H_{n+1}}{\delta u}+2 \bar{i} K_{n+1}(t) x\left(\begin{array}{c}
-r \\
q
\end{array}\right) .
$$

It is remarkable that when $K_{n}(t)=K_{n+1}(t)=0,(23)$ is the Hamiltonian structure of the corresponding isospectral integrable hierarchy of (17). 


\section{Discussion on symmetries and conserved quantities}

In [8], the authors applied the isospectral and nonisospectral integrable AKNS hierarchy to construct $K$ symmetries and $\tau$ symmetries, which constitute an infinite-dimensional Lie algebra. Thus, we also study the $K$ symmetries and $\tau$ symmetries of hierarchy (17) in this section. Moreover, some conserved qualities of hierarchy (17) can be found based on the obtained symmetries. After simple calculations, one can find that $\Phi$ presented in (18) satisfies

$$
\Phi^{\prime}[\Phi f] g-\Phi^{\prime}[\Phi g] f=\Phi\left\{\Phi^{\prime}[f] g-\Phi^{\prime}[g] f\right\}
$$

for $\forall f, g \in S$. Thus, $\Phi$ is the hereditary symmetry of (17). In what follows we can also prove that the following relation holds.

\section{Proposition 1}

$$
\Phi^{\prime}\left[K_{0}\right]=\left[K_{0}^{\prime}, \Phi\right]
$$

where $K_{0}=\left(\begin{array}{c}q_{x} \\ r_{x}\end{array}\right)=u_{t_{0}}$.

In fact,

$$
\Phi^{\prime}\left[K_{0}\right]=\partial\left(\begin{array}{ll}
q_{x} \partial^{-1} q+q \partial^{-1} q_{x} & q_{x} \partial^{-1} r+q \partial^{-1} r_{x} \\
r_{x} \partial^{-1} q+r \partial^{-1} q_{x} & r_{x} \partial^{-1} r+r \partial^{-1} r_{x}
\end{array}\right),
$$

for $\forall f=\left(f_{1}, f_{2}\right)^{T} \in S$, we have

$$
\begin{aligned}
& \Phi^{\prime}\left[K_{0}\right] f=\left(\begin{array}{c}
q_{x x} \partial^{-1} q f_{1}+\left(q^{2}\right)_{x} f_{1}+q_{x} \partial^{-1} q_{x} f_{1}+q_{x x} \partial^{-1} r f_{2}+(q r)_{x} f_{2}+q_{x} \partial^{-1} r_{x} f_{2} \\
r_{x x} \partial^{-1} q f_{1}+(q r)_{x} f_{1}+r_{x} \partial^{-1} q_{x} f_{1}+r_{x x} \partial^{-1} r f_{2}+\left(r^{2}\right)_{x} f_{2}+r_{x} \partial^{-1} r_{x} f_{2}
\end{array}\right), \\
& {\left[K_{0}^{\prime}, \Phi\right]\left(\begin{array}{l}
f_{1} \\
f_{2}
\end{array}\right)} \\
& =K_{0}^{\prime} \Phi\left(\begin{array}{l}
f_{1} \\
f_{2}
\end{array}\right)-\Phi K_{0}^{\prime}\left(\begin{array}{l}
f_{1} \\
f_{2}
\end{array}\right) \\
& =\left(\begin{array}{ll}
\partial & 0 \\
0 & \partial
\end{array}\right) \partial\left(\begin{array}{cc}
q \partial^{-1} q & i+q \partial^{-1} r \\
-i+r \partial^{-1} q & r \partial^{-1} r
\end{array}\right)\left(\begin{array}{l}
f_{1} \\
f_{2}
\end{array}\right)-\Phi\left(\begin{array}{l}
f_{1 x} \\
f_{2 x}
\end{array}\right) \\
& =\left(\begin{array}{c}
q_{x x} \partial^{-1} q f_{1}+3 q q_{x} f_{1}-q_{x} \partial^{-1} q \partial f_{1}+q_{x x} \partial^{-1} r f_{2}+q_{x} r f_{2}+(q r)_{x} f_{2}-q_{x} \partial^{-1} r f_{2 x} \\
r_{x x} \partial^{-1} q f_{1}+r_{x} q f_{1}+q_{x} r f_{1}-r_{x} \partial^{-1} q \partial f_{1}+q r_{x} f_{1}+r_{x x} \partial^{-1} r f_{2}+3 r r_{x} f_{2}-r_{x} \partial^{-1} r f_{2 x}
\end{array}\right) .
\end{aligned}
$$

We therefore verified that (24) is correct. It follows that we can get the following equation because $\Phi$ is a hereditary symmetry:

$$
\Phi^{\prime}\left[K_{m}\right]=\left[K_{m}^{\prime}, \Phi\right]
$$

which means that $\Phi$ is a strong symmetry, where $K_{m}=\Phi^{m}\left(\begin{array}{l}q_{x} \\ r_{x}\end{array}\right)$. 


\section{Proposition 2}

$$
\Phi^{\prime}[x u]+\Phi(x u)^{\prime}-(x u)^{\prime} \Phi=H I
$$

where $u=\left(\begin{array}{c}q_{x} \\ r_{x}\end{array}\right), H=\left(\begin{array}{cc}0 & \bar{i} \partial \\ -\bar{i} \partial & 0\end{array}\right)$, and I is an identity matrix.

In fact,

$$
\Phi^{\prime}[x u]=\left(\begin{array}{ll}
A & B \\
C & D
\end{array}\right)
$$

where

$$
\begin{aligned}
& \left\{\begin{array}{l}
A=q_{x} \partial^{-1} q+x q_{x x} \partial^{-1} q+2 x q_{x} q+q_{x} \partial^{-1} x q_{x}, \\
B=q_{x} \partial^{-1} r+x q_{x x} \partial^{-1} r+x q_{x} r+x q r_{x}+q_{x} \partial^{-1} x r_{x}, \\
C=r_{x} \partial^{-1} q+x r_{x x} \partial^{-1} q+x r_{x} q+x r q_{x}+r_{x} \partial^{-1} x q_{x}, \\
D=r_{x} \partial^{-1} r+x r_{x x} \partial^{-1} r+2 x r_{x} r+r_{x} \partial^{-1} x r_{x} .
\end{array}\right. \\
& \Phi(x u)^{\prime}=\left(\begin{array}{cc}
x q^{2} \partial+x q q_{x}-q_{x} \partial^{-1}\left(q+x q_{x}\right) & x q r \partial+\bar{i} \partial+\bar{i} x \partial^{2}+x r q_{x}-q_{x} \partial^{-1}\left(r+x r_{x}\right) \\
x q r \partial-\bar{i} \partial-\bar{i} x \partial^{2}+x q r_{x}-r_{x} \partial^{-1}\left(q+x q_{x}\right) & x r^{2} \partial+x r r_{x}-r_{x} \partial^{-1}\left(r+x r_{x}\right)
\end{array}\right), \\
& (x u)^{\prime} \Phi=\left(\begin{array}{cc}
x q_{x x} \partial^{-1} q+3 x q q_{x}+x q^{2} \partial & \bar{i} x \partial^{2}+x q_{x x} \partial^{-1} r+2 x r q_{x}+x q r_{x}+x q r \partial \\
-\bar{i} x \partial^{2}+x r_{x x} \partial^{-1} q+2 x q r_{x}+x r q_{x}+x q r \partial & x r_{x x} \partial^{-1} r+3 x r r_{x}+x r^{2} \partial
\end{array}\right),
\end{aligned}
$$

where

$$
(x u)^{\prime}[\sigma]=\left.\frac{d}{d \epsilon}\right|_{\epsilon=0}\left(\begin{array}{l}
x\left(q+\epsilon \sigma_{1}\right)_{x} \\
x\left(q+\epsilon \sigma_{2}\right)_{x}
\end{array}\right)=x \partial\left(\begin{array}{l}
\sigma_{1} \\
\sigma_{2}
\end{array}\right) \quad \Longrightarrow \quad(x u)^{\prime}=\left(\begin{array}{cc}
x \partial & 0 \\
0 & x \partial
\end{array}\right) .
$$

We therefore verified that (25) is correct.

\section{Proposition 3}

$$
\left[K_{1}, x u\right]=[\Phi u, x u]=H u+K_{1}
$$

where $u=\left(\begin{array}{c}q_{x} \\ r_{x}\end{array}\right), H=\left(\begin{array}{cc}0 & \bar{i} \partial \\ -\bar{i} \partial & 0\end{array}\right)$, and $K_{1}=\Phi u$.

In fact,

$$
\begin{aligned}
& \Phi u=\left(\begin{array}{c}
\bar{i} r_{x x}+\frac{1}{2} q_{x}\left(q^{2}+r^{2}\right)+q r r_{x}+q^{2} q_{x} \\
-\bar{i} q_{x x}+\frac{1}{2} r_{x}\left(q^{2}+r^{2}\right)+q r q_{x}+r^{2} r_{x}
\end{array}\right) \\
& (\Phi u)^{\prime}=\left(\begin{array}{cc}
\frac{1}{2}\left(q^{2}+r^{2}\right) \partial+3 q q_{x}+q^{2} \partial+r r_{x} & \bar{i} \partial^{2}+q r \partial+(q r)_{x} \\
-\bar{i} \partial^{2}+q r \partial+(q r)_{x} & \frac{1}{2}\left(q^{2}+r^{2}\right) \partial+3 r r_{x}+r^{2} \partial+q q_{x}
\end{array}\right), \\
& (\Phi u)^{\prime}\left(\begin{array}{c}
x q_{x} \\
x r_{x}
\end{array}\right) \\
& =\left(\begin{array}{c}
\frac{1}{2}\left(q^{2}+r^{2}\right) \partial\left(x q_{x}\right)+3 x q q_{x}^{2}+q^{2} \partial\left(x q_{x}\right)+x r r_{x} q_{x}+\bar{i} \partial^{2}\left(x r_{x}\right)+q r \partial\left(x r_{x}\right)+x r_{x}(q r)_{x} \\
-\bar{i} \partial^{2}\left(x q_{x}\right)+q r \partial\left(x q_{x}\right)+x q_{x}(q r)_{x}+\frac{1}{2}\left(q^{2}+r^{2}\right) \partial\left(x r_{x}\right)+3 x r r_{x}^{2}+r^{2} \partial\left(x r_{x}\right)+x r_{x} q q_{x}
\end{array}\right) \text {. }
\end{aligned}
$$


Then we have

$$
\begin{aligned}
& (x u)^{\prime}[\Phi u]=\left(\begin{array}{c}
x \partial\left(\bar{i} r_{x x}+\frac{1}{2} q_{x}\left(q^{2}+r^{2}\right)+q r r_{x}+q^{2} q_{x}\right) \\
x \partial\left(-\bar{i} q_{x x}+\frac{1}{2} r_{x}\left(q^{2}+r^{2}\right)+q r q_{x}+r^{2} r_{x}\right)
\end{array}\right) \\
& {[\Phi u, x u]=(\Phi u)^{\prime}[x u]-(x u)^{\prime}[\Phi u]=\left(\begin{array}{cc}
0 & \bar{i} \partial \\
-\bar{i} \partial & 0
\end{array}\right)\left(\begin{array}{l}
q_{x} \\
r_{x}
\end{array}\right)+K_{1}=H u+K_{1} .}
\end{aligned}
$$

We therefore verified that (26) is correct.

\section{Proposition 4}

$$
\left[K_{m}, K_{n}\right]=0, \quad m, n=0,1,2, \ldots,
$$

where $K_{m}=\Phi^{m} u, K_{n}=\Phi^{n} u$.

\section{Proposition 5}

$$
\left[\Phi^{m} x u, x u\right]=m \Phi^{m-1}(x u) .
$$

The proofs of Proposition 4 and Proposition 5 were presented in [20].

From the above results we can get

$$
\left[\Phi^{m} x u, \Phi^{n} x u\right]=(m-n) \Phi^{m+n-1}(x u), \quad m=0,1,2, \ldots ; n=0,1,2, \ldots
$$

From (26), one can find that $\left\{\Phi^{n} u, \Phi^{m} x u\right\}$ cannot constitute a Lie algebra. However, $\left\{\Phi^{n} u, n=0,1,2, \ldots\right\}$ and $\left\{\Phi^{n} x u, n=0,1,2, \ldots\right\}$ constitute the infinite-dimensional Lie algebra, respectively based on the above analysis.

Next we derive some conserved qualities of Tu isospectral hierarchy

$$
u_{t_{n}}=\left(\begin{array}{l}
q \\
r
\end{array}\right)_{t_{n}}=\Phi^{n}\left(\begin{array}{l}
q_{x} \\
r_{x}
\end{array}\right) \text {. }
$$

Definition $3([11,12,14])$ If we have known the integrable hierarchy $u_{t}=K_{n}(u)$, then $v$ satisfying the following equation

$$
\frac{d v}{d t}+K^{\prime *} v=0
$$

is called the conserved covariance, where $K^{\prime}$ is the linearized operator of $K$, and $K^{\prime *}$ denotes a conjugate operator of $K^{\prime}$.

Proposition 6 ([14]) If $\sigma$ is a symmetry of Eq. $u_{t}=K_{n}(u), v$ is its conserved covariance, then we have

$$
\int_{-\infty}^{\infty} v \sigma d x=\langle v, \sigma\rangle,
$$

which is independent of time $t$, that is, $\frac{d}{d t}\langle v, \sigma\rangle=0$. 
Definition $4([11,12,14])$ If $F^{\prime} f=\langle v, f\rangle$ for $\forall f \in S$, then $v$ is called the gradient of the functional $F$, which is denoted by $v=\frac{\delta F}{\delta u}$.

Proposition 7 ([14]) If $v^{\prime}=v^{\prime *}$, then $v$ is the gradient of the following functional:

$$
F=\int_{0}^{1}\langle v(\lambda u), u\rangle d \lambda
$$

According to the symbols above, we can deduce the following.

Proposition $8([11,12])$ If I is a conserved quality of the hierarchy $u_{t}=K_{n}(u)$, and the conserved covariance $v$ satisfies

$$
I^{\prime} K_{n}=\left\langle v, K_{n}\right\rangle,
$$

then one obtains

$$
\frac{\partial I}{\partial t}+\left\langle v, K_{n}\right\rangle=0
$$

that is,

$$
\frac{\partial v}{\partial t}+K_{n}^{\prime *} v+v^{\prime} K_{n}=0
$$

Hence, we derive the following conserved qualities related to the integrable hierarchy $u_{t}=K_{n}(u)$ :

$$
I_{m}=\int_{0}^{1}\left\langle\partial_{x}^{-1} K_{m}(\lambda u), u\right\rangle d \lambda
$$

In addition, a few conserved qualities are also derived for the integrable hierarchy (28) as follows:

$$
I_{0}=\int_{0}^{1}\left\langle\partial_{x}^{-1} K_{0}(\lambda u), u\right\rangle d \lambda=\int_{0}^{1}\left\langle\left[\left(\begin{array}{cc}
0 & -1 \\
1 & 0
\end{array}\right)\left(\begin{array}{l}
q_{x} \lambda \\
r_{x} \lambda
\end{array}\right)\right]^{T},\left(\begin{array}{l}
q \\
r
\end{array}\right)\right\rangle d \lambda=\int_{-\infty}^{\infty}\left(q_{x} r-r_{x} q\right) d x,
$$

where

$$
K_{0}=\Phi^{0} u=\left(\begin{array}{l}
q_{x} \\
r_{x}
\end{array}\right)=\left(\begin{array}{cc}
0 & 1 \\
-1 & 0
\end{array}\right)\left(\begin{array}{c}
-r_{x} \\
q_{x}
\end{array}\right)
$$

Moreover, we have

$$
\begin{aligned}
K_{1} & =\Phi u=\left(\begin{array}{c}
\bar{i} r_{x x}+\frac{1}{2} q_{x}\left(q^{2}+r^{2}\right)+q r r_{x}+q^{2} q_{x} \\
-\bar{i} q_{x x}+\frac{1}{2} r_{x}\left(q^{2}+r^{2}\right)+q r q_{x}+r^{2} r_{x}
\end{array}\right) \\
& =\left(\begin{array}{cc}
0 & 1 \\
-1 & 0
\end{array}\right)\left(\begin{array}{l}
\bar{i} q_{x x}-\frac{1}{2} r_{x}\left(q^{2}+r^{2}\right)-q r q_{x}-r^{2} r_{x} \\
\bar{i} r_{x x}+\frac{1}{2} q_{x}\left(q^{2}+r^{2}\right)+q r r_{x}+q^{2} q_{x}
\end{array}\right),
\end{aligned}
$$




$$
\begin{aligned}
I_{1} & =\int_{0}^{1}\left\langle\left[\left(\begin{array}{cc}
0 & -1 \\
1 & 0
\end{array}\right)\left(\begin{array}{c}
\bar{i} r_{x x} \lambda+\frac{1}{2} q_{x}\left(q^{2}+r^{2}\right) \lambda^{3}+q r r_{x} \lambda^{3}+q^{2} q_{x} \lambda^{3} \\
-\bar{i} q_{x x} \lambda+\frac{1}{2} r_{x}\left(q^{2}+r^{2}\right) \lambda^{3}+q r q_{x} \lambda^{3}+r^{2} r_{x} \lambda^{3}
\end{array}\right)\right]^{T},\left(\begin{array}{l}
q \\
r
\end{array}\right)\right\rangle d \lambda \\
& =\int_{-\infty}^{\infty}\left[\bar{i} \frac{1}{2}\left(q q_{x x}+r r_{x x}\right)+\frac{1}{8}\left(q^{2}+r^{2}\right)\left(q_{x} r-r_{x} q\right)\right] d x, \\
\vdots & \\
I_{k} & =\int_{-\infty}^{\infty}\left\langle\left(\begin{array}{cc}
0 & -1 \\
1 & 0
\end{array}\right) K_{k}(\lambda u),\left(\begin{array}{l}
q \\
r
\end{array}\right)\right\rangle d \lambda .
\end{aligned}
$$

\section{Acknowledgements}

Not applicable.

\section{Funding}

This work was supported by the National Natural Science Foundation of China (grant No. 11971475).

\section{Availability of data and materials}

Not applicable.

\section{Ethics approval and consent to participate}

Not applicable.

\section{Competing interests}

The authors declare that they have no competing interests.

\section{Consent for publication}

Not applicable.

\section{Authors' contributions}

The two authors contributed equally to the manuscript and typed, read, and approved the final manuscript.

\section{Publisher's Note}

Springer Nature remains neutral with regard to jurisdictional claims in published maps and institutional affiliations.

Received: 5 January 2020 Accepted: 23 March 2020 Published online: 22 April 2020

\section{References}

1. Magri, F.: Nonlinear Evolution Equations and Dynamical Systems. Springer Lecture Notes in Physics, vol. 120, p. 233. Springer, Berlin (1980)

2. Ablowitz, M.J., Segur, H.: Solitons and the Inverse Scattering Transform. SIAM, Philadelphia (1981)

3. Newell, A.C.: Solitons in Mathematics and Physics. SIAM, Philadelphia (1985)

4. Tu, G.Z.: The trace identity, a powerful tool for constructing the Hamiltonian structure of integrable systems. J. Math. Phys. 30, 330-338 (1989)

5. Ma, W.X.: A new hierarchy of Liouville integrable generalized Hamiltonian equations and its reduction. Chin. J. Contemp. Math. 13(1), 79 (1992)

6. Qiao, Z.J.: Generation of soliton hierarchy and general structure of its commutator representations. Acta Math. Appl. Sin. 18(2), 287-301 (1995)

7. Li, Y.S.: A kind of evolution equations and the deform of spectral. Sci. Sin., Ser. A 25, 385-387 (1982) (in Chinese)

8. Li, Y.S., Zhu, G.C.: New set of symmetries of the integrable equations, Lie algebras and non-isospectral evolution equations: II. AKNS suystem. J. Phys. A, Math. Gen. 19, 3713-3725 (1986)

9. Kaup, D.J., Newell, A.C.: An exact solution for a derivative nonlinear Schrödinger equation. J. Math. Phys. 19(4), 798-804 (1978)

10. Li, Y.S., Zhuang, D.W.: Nonlinear evolution equations related to characteristic problems dependent on potential energy. Acta Math. Sin. 25(4), 464-474 (1982) (in Chinese)

11. Zhang, Y.F., Tam, H.: A few integrable systems and spatial spectral transformations. Commun. Nonlinear Sci. Numer. Simul. 14(11), 3770-3783 (2009)

12. Zhang, Y.F., Rui, W.J.: A few continuous and discrete dynamical systems. Rep. Math. Phys. 78(1), 19-32 (2016)

13. Chang, X.K., Hu, X.B., Li, S.H.: Moment modification, multipeakons, and nonisospectral generalizations. J. Differ. Equ. 265(9), 3858-3887 (2018)

14. Ma, W.X.: K symmetries and $\tau$ symmetries of evolution equations and their Lie algebras. J. Phys. A, Math. Gen. 23, $2707-2716(1990)$

15. Fan, E.: The integrability of nonisospectral and variable-coefficient KdV equation with binary Bell polynomials. Phys. Lett. A 375(3), 493-497 (2011)

16. Ma, W.X.: An approach for constructing non-isospectral hierarchies of evolution equations. J. Phys. A, Math. Gen. 25, L719-L726 (1992) 
17. Ma, W.X.: A simple scheme for generating nonisospectral flows from the zero curvature representation. Phys. Lett. A 179, 179-185 (1993)

18. Qiao, Z.J.: New hierarchies of isospectral and non-isospectral integrable NLEEs derived from the Harry-Dym spectral problem. Physica A 252, 377-387 (1998)

19. Zhang, Y.F., Mei, J.Q., Guan, H.Y.: A method for generating isospectral and nonisospectral hierarchies of equations as well as symmetries. J. Geom. Phys. 147(103538), 1-15 (2020)

20. Zhang, Y.F., Wang, H.F., Bai, N.: A general method for generating nonisospectral integrable hierarchies by the use of loop algebras. Preprint

21. Yu, F.J.: A novel non-isospectral hierarchy and soliton wave dynamics for a parity-time-symmetric nonlocal vector nonlinear Gross-Pitaevskii equations. Commun. Nonlinear Sci. Numer. Simul. 78(104852), 1-18 (2019)

22. Gao, X.D., Zhang, S.: Inverse scattering transform for a new non-isospectral integrable non-linear AKNS model. Therm. Sci. 21(1), S153-S160 (2017)

23. Estévz, P.G., Savdón, C.: Miura-reciprocal transformations for non-isospectral Camassa-Holm hierarchies in $2+1$ dimensions. J. Nonlinear Math. Phys. 20(4), 552-564 (2013)

24. Estévz, P.G. Lejarreta, J.D., Sardón, C.: Non-isospectral $1+1$ hierarchies arising from a Camassa-Holm hierarchy in $2+1$ dimensions. J. Nonlinear Math. Phys. 18(1), 9-28 (2011)

25. Zhao, X.H., Tiao, B., Li, H.M., Guo, Y.J.: Solitons, periodic waves, breathers and integrability for a nonisospectral and variable-coefficient fifth-order Korteweg-de Vries equation in fluids. Appl. Math. Lett. 65, 48-55 (2017)

26. Wang, H.F., Li, C.Z.: Affine Weyl group symmetries of Frobenius Painlevé equations. Math. Methods Appl. Sci. 43 3238-3252 (2020)

\section{Submit your manuscript to a SpringerOpen ${ }^{\circ}$ journal and benefit from:}

- Convenient online submission

- Rigorous peer review

- Open access: articles freely available online

- High visibility within the field

- Retaining the copyright to your article

Submit your next manuscript at $\boldsymbol{~ s p r i n g e r o p e n . c o m ~}$ 